\title{
NEAR STALL FLOW ANALYSIS IN THE TRANSONIC FAN OF THE RTA PROPULSION SYSTEM
}

\author{
Chunill Hah, ${ }^{1}$ \\ NASA Glenn Research Center, Cleveland, $\mathrm{OH} 44135$
}

\begin{abstract}
Turbine-based propulsion systems for access to space have been investigated at NASA Glenn Research center. A ground demonstrator engine for validation testing has been developed as a part of the program. The demonstrator, the Revolutionary Turbine Accelerator (RTA-1), is a variable cycle turbofan ramjet designed to transition from an augmented turbofan to a ramjet that produces the thrust required to accelerate the vehicle to Mach 4 . The RTA- 1 is designed to accommodate a large variation in bypass ratio from sea level static to Mach 4 flight condition. A key component of this engine is a new fan stage that accommodates these large variations in bypass ratio and flow ranges. In the present study, unsteady flow behavior in the fan of the RTA-1 is studied in detail with large eddy simulation (LES) and the numerical results are compared with measured data. During the experimental study of the fan stage, humming sound was detected at $100 \%$ speed near stall operation. The main purpose of the study is to investigate details of the unsteady flow behavior at near stall operation and to identify a possible cause of the hum. The large eddy simulation of the current flow field reproduces main features of the measured flow very well. The LES simulation indicates that non-synchronous flow instability develops as the fan operates toward the stall limit. The FFT analysis of the calculated wall pressure shows that the rotating flow instability has the characteristic frequency that is about $50 \%$ of the blade passing frequency.
\end{abstract}

\footnotetext{
${ }^{1}$ Scientist, Turbomachinery and Heat Transfer Branch, Aeropropulsion Division, M.S. 5-10
} 


\section{Introduction}

The revolutionary Turbine Accelerator Project (RTA) at NASA was to develop turbine-based propulsion system for access to the space. Turbine based combined cycle (TBCC) propulsion can provide an aircraft-like space launch operation that can potentially reduce the launch cost and improve safety. The proposed system requires high Mach number turbine engines to accelerate the vehicle to scramjet takeover speed. Developing a turbine accelerator with Mach number 4+ capabilities and a scramjet with low ignition speed $(M<4)$ to enable transition from the low speed to high speed propulsion system are two main challenges for the program. A ground demonstrator, RTA-1, was developed for a Mach 4 turbine engine which leverages existing hardware assets and utilizing slave cooling system. The demonstrator (RTA-1) is a variable cycle, turbine ramjet designed to transition from an augmented turbofan to a ramjet that produces the thrust required to accelerate the vehicle from ground take-off to Mach 4.

The primary goal of the fan stage design was to provide a high pressure ratio level with good efficiency at takeoff through the mid range of engine operation while avoiding stall and losses at the high flight Mach numbers, without the use of variable inlet guide vanes. The fan stage was designed by GE and tested at NASA Glenn Research Center. During the test, humming sound from the fan was detected when the fan was operated at near stall, $100 \%$ design speed. The primary focus of the current paper is to investigate the characteristics of the fan flow field at near stall operation. A possible flow instability at near stall operation is investigated with the numerical simulation using a Large Eddy Simulation.

Various studies have been reported to understand the flow instability (for example, Mailach [2001], Maerz et al. [2002], Kielb et al. [2003], and Inoue et al. [2004]). Rotating flow instability is a phenomenon that occurs in the tip flow region of axial fans and compressors during stable operation. Spectral analysis of the unsteady casing pressure shows characteristic frequencies of the flow instability. For subsonic compressors, the phenomenon can often be observed in highly staggered compressor rotors with relatively large tip clearance when operated near the stall limit. The phenomenon in high speed compressors is not yet well understood. The flow field near the casing in a transonic compressor is very complex. Dominant features of the compressor endwall flow include the tip clearance flow; interactions among the tip clearance flow, the passage shock, and the endwall boundary layers; and accumulation of low momentum fluid due to radial migration. In the following sections, results from the LES simulations will be first compared with the available measured data on the RTA-1 fan and possible flow instability in the current fan is examined in detail.

\section{Test Fan Rotor}

The fan stage was designed to operate over a large range of operating conditions, much larger than in more typical turbomachinery applications. The fan rotor was designed to produce a high pressure ratio at lower flight Mach numbers while maintaining a good stall margin across wide range of operating conditions. In order to achieve the required performance, a forward swept fan rotor design was applied.

The overall engine and three-dimensional airfoil geometry is shown in Figure 1. Details of the design of the fan stage are given by Mielke [2010] and Mielke et al. [2010]. Test procedure and aerodynamic test of the fan stage are given by Suder et al. [2005]. For the current study, flow fields with the smooth casing at $100 \%$ design speed are investigated.

\section{Numerical Procedure}

A Large Eddy Simulation (LES) method (Hah et al. [2008]) was applied in the present study to accurately capture unsteady interactions between the tip clearance vortex and the passage shock. With spatially-filtered Navier-Stokes equations, the subgrid-scale stress tensor term must be modeled properly for closure of the governing equations. A Smagorinsky-type eddy-viscosity model was used for the subgrid stress tensor, and the standard dynamic model by Germano et al. [1991] was applied. 
In the current study, the governing equations are solved with a pressure-based implicit method using a fully conservative control volume approach. A third-order accurate interpolation scheme is used for the discretization of convection terms and central differencing is used for the diffusion terms. The method is of second-order accuracy with smoothly varying grids. For the timedependent terms, an implicit second-order scheme is used and a number of sub-iterations are performed at each time step.

The computational grid for a single blade passage consists of 98 nodes in the blade-to-blade direction, 78 nodes in the spanwise direction, and 280 nodes streamwise direction. The rotor tip clearance geometry is accurately represented by 20 nodes in the blade-to-blade direction, 14 nodes in the spanwise direction, and 140 nodes in the streamwise direction. The I-grid topology is used to reduce grid skewness and a single-block grid is used. The same grid size is used for both the single passage analysis and the full annulus analysis.

Standard boundary conditions for an isolated rotor were applied at the boundaries of the computational domain. The inflow boundary of the computational domain was located 6 average blade heights upstream of the rotor leading edge in order to damp out any possible reflections. Circumferentially averaged static pressure at the casing was specified to control the mass flow rate. Non-reflecting boundary conditions were applied at the inlet and the exit boundaries. For the current study, isolated fan configuration is analyzed as the rotating instability occurs near the rotor tip section. Any possible upstream effects of the outlet guide vane is not included in the analysis.

All the computations were performed with NASA's Columbia supercomputer system, which allows parallel computation with up to 512 processors.

\section{Overall Flow Structure}

Aero test was performed at the Single Stage Axial Compressor Facility, NASA Glenn Research Center. Figure 2 shows schematic of the instrumentation (Suder et al. [2005]). Measured and calculated total pressure rise across the fan rotor are compared in Figure 3. Total pressure rise across the fan stage is also given in Figure 3. The measured total pressure rise across fan was obtained from the leading edge instrumentations on the outlet guide vane. The averaged flow fields from the LES simulations were obtained by averaging flow field over 2000 time steps. The results from the numerical simulations are given at choke (point A), near design (point B), near stall (point C), and close to the stall (point D) in Figure 3. The simulation point E is estimated stall point from the numerical simulation. The time-averaged LES solutions reproduce the measured pressure rise across fan rotor qualitatively well. The steady Reynolds-averaged Navier-Stokes procedure with the standard two-equation model was also applied for the current fan rotor field. The RANS calculates the overall pressure rise about $6 \%$ higher for this fan. Time-averaged casing static pressure distributions are compared in Figure 4 at near choke, design and near stall operation. Calculated pressure distributions agree well with the measured values at all three operating conditions. Effects of the interaction between the tip clearance vortex and the passage shock are clearly shown on the time-averaged casing static pressure distribution. Comparisons shown in Figures 3 and 4 indicate that the currently applied LES reproduces the flow field correctly. In the following sections, detailed flow features at the near stall operation are examined first with the LES results based on a single passage simulation. Then unsteady flow features at near stall are further investigated with the LES results based on full annulus simulation.

\section{Flow unsteadiness due to interaction between tip clearance vortex and passage shock at near stall operation}

When the transonic compressor operates toward stall limit, interaction between the tip clearance vortex and the passage shock becomes stronger. The shock and vortex interaction is inherently unsteady (Thomer et al.[2002], Hah et al.[2008]) and the flow becomes transient when a transonic compressor operates at near stall condition. Additionally shed vortices from the trailing edge can interact with the tip clearance vortex and further increases the transient nature of the flow 
field at near stall operation. To assess transient nature of the flow field a LES simulation with a single blade passage was performed first. Instantaneous pressure distributions at the blade tip section at near stall condition are shown in Figure 5. In Figure 5, instantaneous distributions are given at three equally spaced times during which the rotor moves one blade passage. In figure 5, both trajectory of tip clearance core vortex and the location of the passage shock are clearly shown and changes in the flow field due to their interaction are visible. Instantaneous axial velocity distributions are given in Figure 6. Instantaneous velocity vectors near the leading edge are given in Figure 7. The LES simulation shows that the incidence angle changes up to 7 degrees at this near stall operation. Due to the blade design, the tip core vortex stays well inside the blade passage at near the stall limit and it appears that the tip clearance core vortex will not move forward to the leading edge plane even the short-length scale stall inception initiates. Instantaneous velocity vectors given in Figure 7 shows that flow does not spill over from one blade passage into the adjacent blade passage at this near stall condition with the single passage flow simulation. One interesting observation in Figure 5 is that the passage shock is still attached to the leading edge and stays inside the blade passage. According to the results in figures 5,6 and 7, The flow field at the near stall operation of the current fan design shows many benefits of advanced forward swept rotor design, passage shock staying inside blade passage, tip leakage core vortex staying close to the suction side of the blade, and reduced unsteadiness at near stall condition. Figure 8 shows an instantaneous pressure distribution at $40 \%$ span at near stall operation (point D in Figure 3). Instantaneous pressure distribution in Figure 8 shows vortex shedding clearly. At this operating condition, vortex shedding initiates at the blade suction surface where the passage shock interacts with the blade boundary layer. Results of Fast Fourier Transformation (FFT) of the calculated casing static pressure at $5 \%$ chord downstream from the leading edge are shown in Figure 9. At this location, characteristic frequency is more clearly shown. The blade passing frequency (BPF) is $7200 \mathrm{~Hz}$ at $100 \%$ design rotor speed. The FFT results in Figure 9 show a dominant frequency component at $3750 \mathrm{~Hz}$, which is $52 \%$ of BPF. Instantaneous flow fields were examined in detail to identify the origin of the dominant frequency shown in Figure 9. At this operating condition, the LES simulation shows that the vortex shedding frequency and the frequency of the tip leakage vortex oscillation is very close and the $3750 \mathrm{~Hz}$ represents both the vortex shedding and the tip clearance vortex oscillation. Vortex shedding from the blade can not be periodic even if the blade geometry is identical with the uniform incoming flow. Also tip clearance vortex oscillation is not periodic over the full annulus if the fan is operated toward the stall limit (Hah et al. [2008]). However the results from a single blade passage simulation provide some aspect of flow field at various operating conditions.

\section{Rotating flow instability and humming sound at near stall operation}

To calculate unsteady flow fields at near stall condition (operating point D in Figure 3), Large Eddy Simulation was applied over the full annulus. Figure 10 shows an instantaneous distribution of static pressure at the blade tip section. The instantaneous pressure distribution shows that the flow field varies significantly among blade passages. Several regions with large blockage are formed. The large blockage region spans over several consecutive blade passages. At this operating condition, blade passages with large blockage are about half of the total blade passages. FFT results of the static pressure on the suction surface at $5 \%$ downstream from the leading edge, rotor tip are given in Figure 11. The pressure spectrum in Figure 11 is quite different from the one from the single passage simulation. The overall level is much higher compared to the results from a single passage analysis. Power spectrum in Figure 11 shows one peak at $147 \mathrm{~Hz}$ and a peak region around 3200 to $5000 \mathrm{~Hz}$. Both frequencies are not synchronous to the fan operation. The $147 \mathrm{~Hz}$ corresponds about $50 \%$ of the rotor rotation frequency. This low characteristic frequency has been observed in other transonic compressors at near stall operation (Spakvszky et al.[1999], and Gannon and Hobson [2007]). This frequency is due to the rotation of the region with large blockage shown in Figure 10. The same phenomenon was also observed recently in a radial transonic rotor and the mechanism was explained by Hah et al. [2008].

The peak area, around 3200 to $5200 \mathrm{~Hz}$ in Figure 11, represents a rotating flow instability which is often observed in subsonic compressors at near stall. This frequency domain of rotating flow instability is less pronounced than those from a radial transonic rotor as the flow field near the 
rotor tip is smoother due to the forward-swept design. Figure 12 shows changes in static pressure at blade tip over several blade passages. Changes in velocity vectors on the same blade passages are given in Figure 13. Instantaneous velocity vectors in Figure 13 shows that flow spillage into the adjacent blade passage over the leading edge at several blade passages. This leading edge flow spillage occurs rather randomly in time and roughly half of the blade passages show flow spillage at a given time. The flow spillage at the leading edge does not occur over the entire annulus at any given time. For a given blade passage, healthy flow pattern without excessive flow incidence and resulting flow spillage returns after periodically. This enables the rotor to operate in a stable mode. This large change in flow pattern and resulting changes in pressure and vortex shedding at the leading edge seems to be the main cause of the peak area at around $4000 \mathrm{~Hz}$ in Figure 12. A humming sound was detected when the current fan was operated at the near stall condition D. The peak area in the frequency around $4000 \mathrm{~Hz}$ represents rotating flow instability and is thought to be cause of the hum. Similar whistling sound was detected when a subsonic compressor was operated with rotating flow instability at near stall (Martz et al. [2003]).

\section{Concluding Remarks}

Unsteady flow behavior in the fan rotor of the RTA-1 system at near stall condition is studied in detail with LES. The calculated flow field agrees fairly well with available measured data. The detailed examination of the simulated flow field shows that the flow becomes unsteady at near stall condition due to interaction between the tip clearance vortex and the passage shock. Flow spills over into the adjacent blade passage intermittently at near stall operation. At near stall operation, flow structure varies significantly among blade passages and change in flow pattern is random. At near stall, about half of the blade passages show flow spillage at the leading edge due to excessive flow incidence at given time. For a specific blade passages, healthy and unhealthy flow pattern repeats in time at near stall. This large change in flow structure produces vortex shedding at the leading edge and large variation in pressure field. The FFT results from the simulated pressure field shows rotating flow instability at near stall and the humming sound from the fan at near stall operation could be caused by this rotating flow instability. Further investigations are planned to identify other possible causes of the hum. Detailed acoustic simulations for the hum will be conducted in the future.

\section{Acknowledgment}

The author would like to acknowledge various helpful discussions about the overall program and details of the rig test from K.L. Suder and P.S. Prahst of NASA Glenn Research Center.

\section{References}

1. Gannon, A.J., and Hobson, G.V., 2009, "Pre-Stall Instability Distribution over a Transonic Compressor Rotor," ASME Journal of Fluid Engineering, Vol.131,pp.1-11..

2. Germano, M., Piomelli, U., Moin, P., and Cabot, W. H., 1991, "A Dynamic Subgrid-Scale EddyViscosity Model," Journal of Fluid Mechanics, Vol. A3, pp. 170-176.

3. Hah, C., Bergner, J., Schiffer, H.P., 2008, "Tip Clearnace Vortec Oscillation, Vortex Shedding and Rotating Instabilities in an Axial Transonic Compressor Rotor," ASME Paper GT2008-50105.

4. Inoue, M., Kuromaru, M, Yoshida, S., Minami, T., Yamada, K., and Furukawa, M., 2004, "Effects of Tip Clearance on Stall Evolution Process in a Low-Speed Axial Compressor Stage," ASME Paper GT2004-5335.

5. Kielb, R. E., Barter, J. W., Thomas, J., and Hall, K. C., 2003, "Blade Excitation by Aerodynamic Instabilities-A Compressor Blade Study,” ASME Paper GT2003-38634. 
6. Maerz, J., Hah, C., and Neise, W, 2002, "An Experimental and Numerical Investigation into the Mechanism of Rotating Instability," ASME Journal of Turbomachinery, Vol. 124, pp. 367-375.

7. Mailach, R., Lehmann, I., and Vogeler, K., 2000, "Rotating Instabilities in an Axial Compressor Originating from the Fluctuating Blade Tip Vortex," ASME Paper 2000-GT.

8. Mielke, M., Clark, D., and Wood, P., 2009,"RTA/GE57 Subscale Fan Rig, Final Report - Aero \& Aeromechanical," NASA CR-2010-215815.

9. Mielke, M., 2009,"RTA/GE57 Subscale Fan Rig, Final Report - Mechanical Design," NASA CR2010-215814.

10. Spakovszky, Z.S., Weigl, H.J., Paduano, J.D.,Schalkqwyk, C.M., Suder, K.L., Bright, M., and Strazisar, A.J., 1999, "Rotating Stall Control in aHigh-Speed Stage with Inlet Distortion, Part 1 Radial Distortion", ASME Journal of Turbomachinery, Vol.121,pp.510-516.

11. Suder, K.L., Adamcyzk,J.A.,Wood, P., 2005, "Design and Analysis of a Mach 4 Capable Fan Stage for RTA Turbine Based Combined Cycle Propulsion System", JANNAF(Joint Army, Navy, NASA,Air Force) $42^{\text {nd }}$ CS/30thAPS/24thPSHSJoint Meeting, Charleston, SC June, 2005.

12. Thomer, O., Klass, M., Schroeder, W., and Krause, E., 2002, "Interaction between Longitudinal Vortices and Normal and Oblique Shocks," Proceedings of Fifth World Congress on Computational Mechanics.
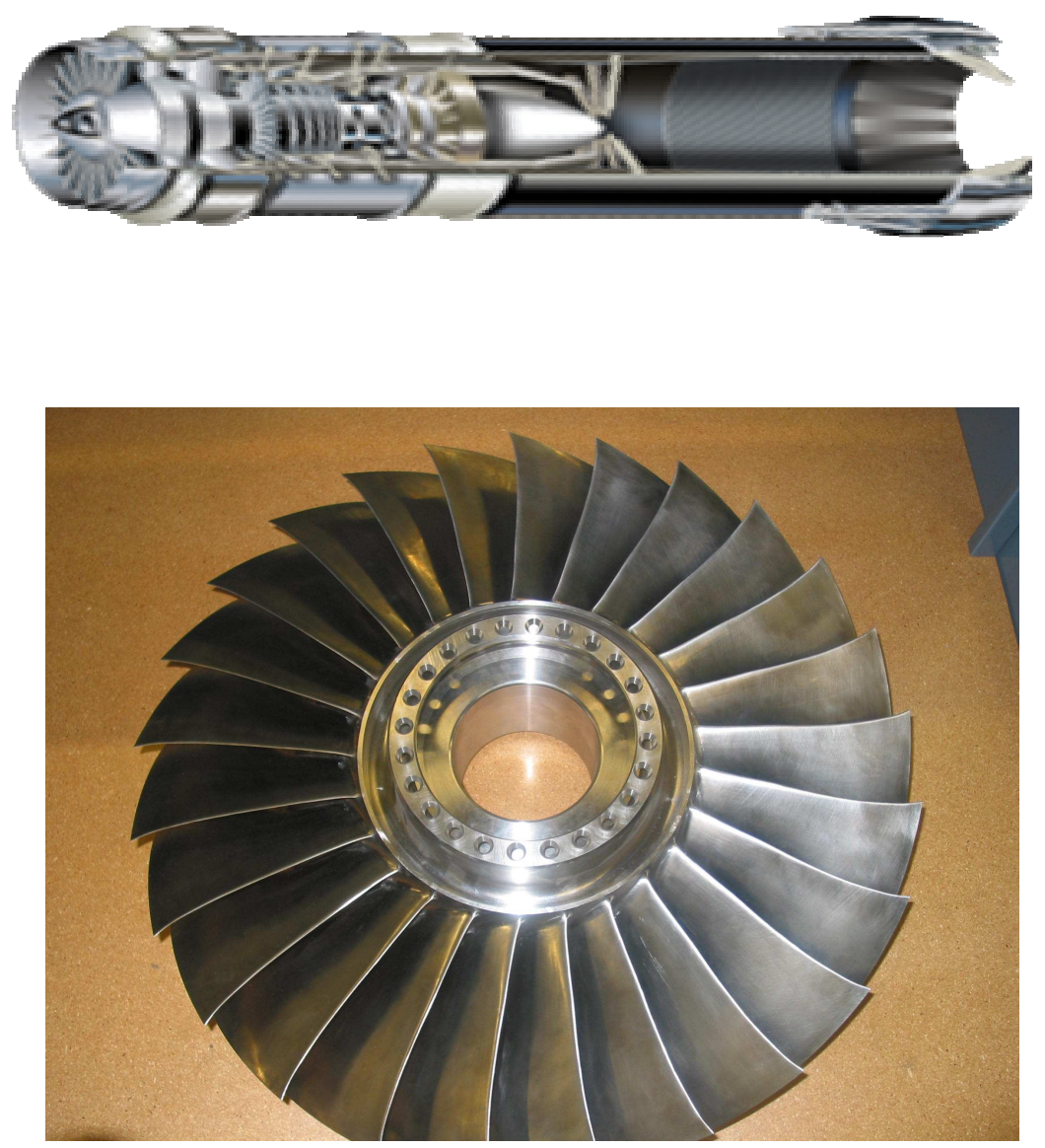

Figure 1: RTA-1 engine and fan blisk. 


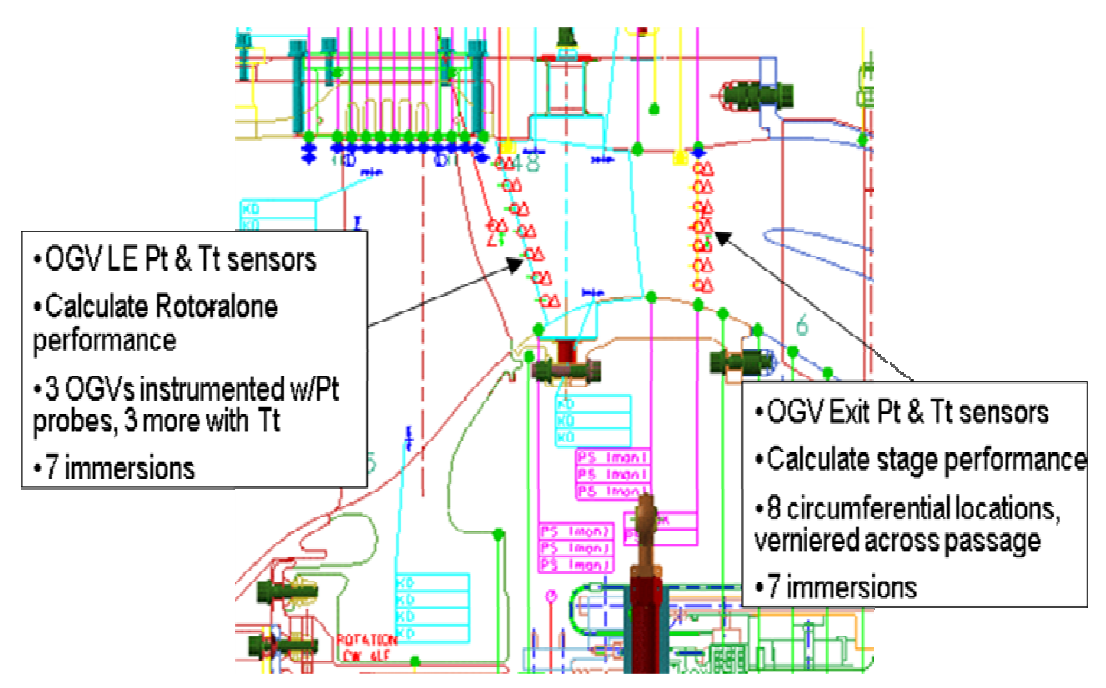

Figure 2: Fan stage instrumentation.

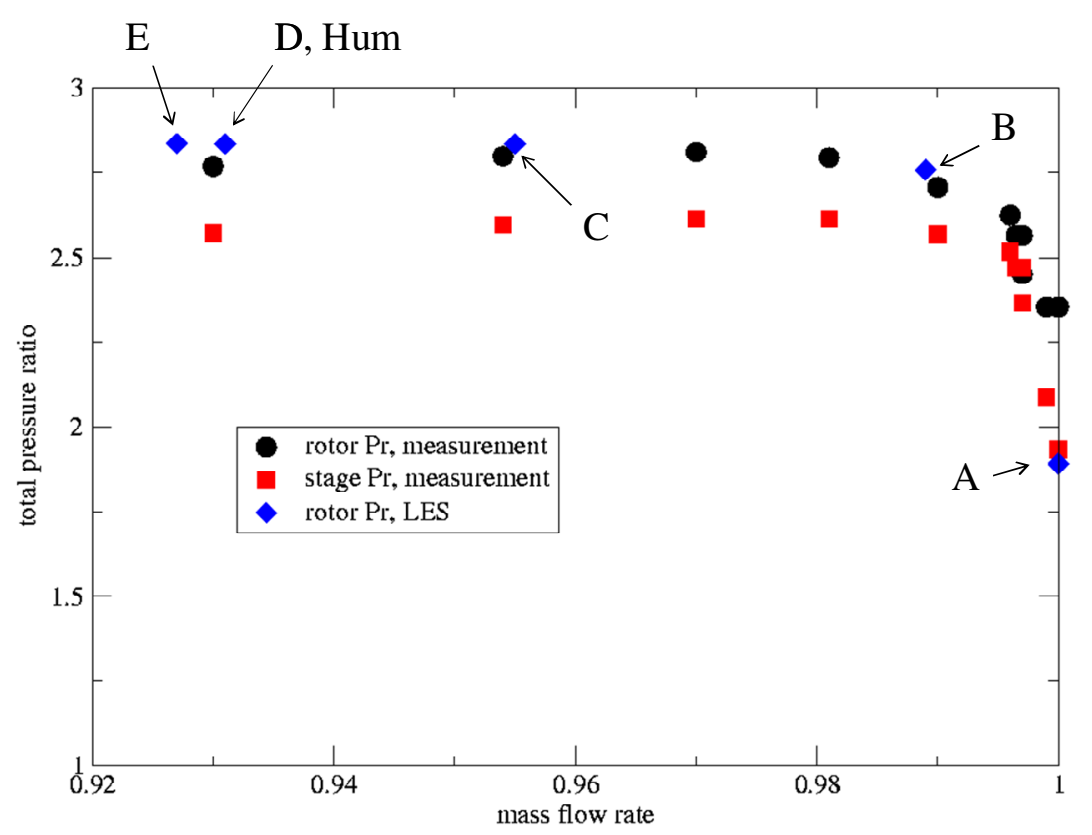

Figure 3: Comparison of pressure rise characteristics of the rotor at design rotor speed. 


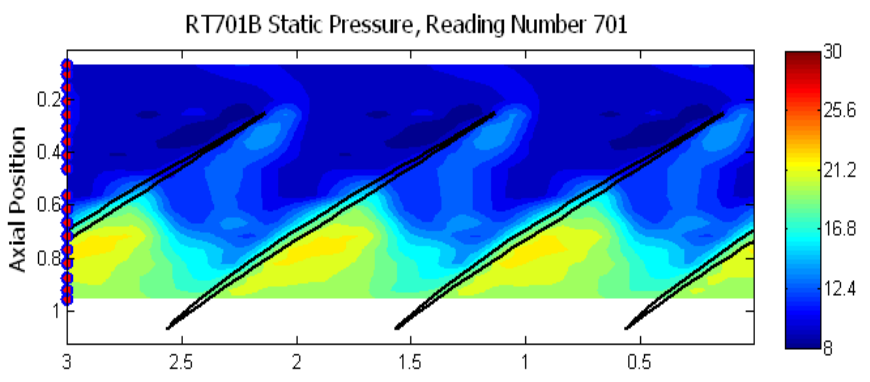

Measurement

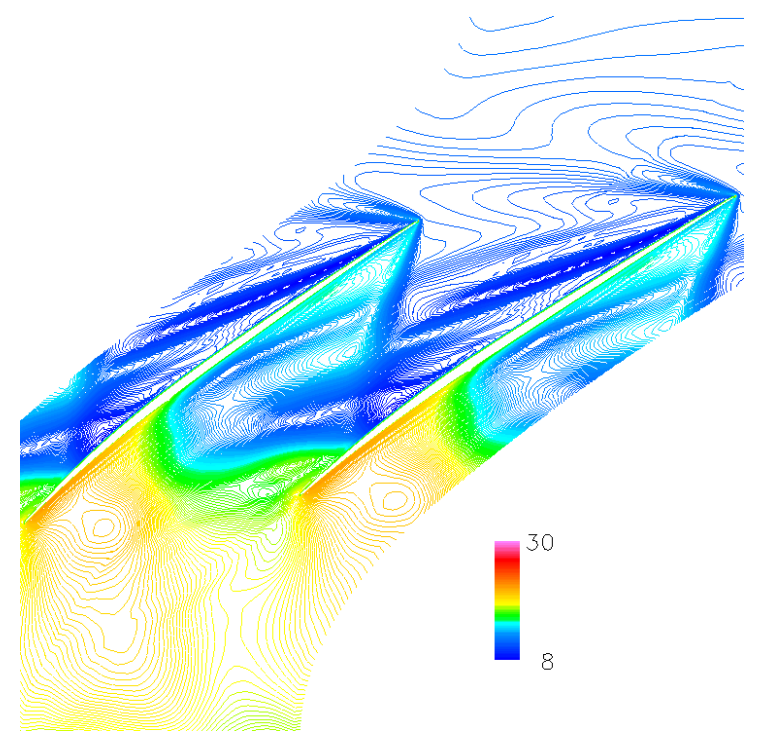

\section{LES}

Figure 4a. Comparisons of casing static pressure at choke (Point A in Figure 3).

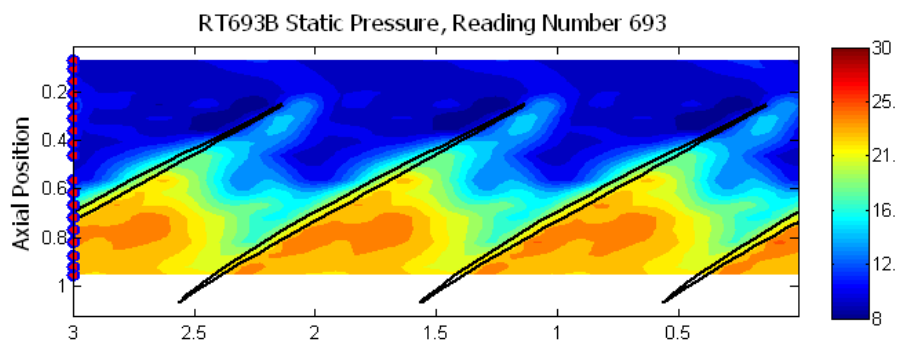

Measurement

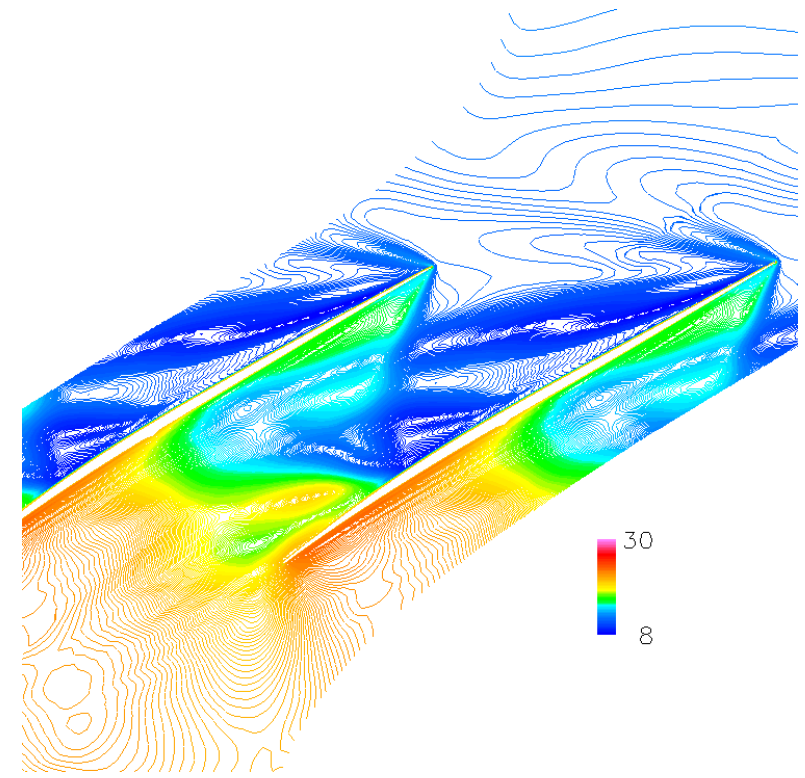

\section{LES}

Figure 4b: Comparison of casing static pressure distribution at design condition (Point B in Figure 3). 


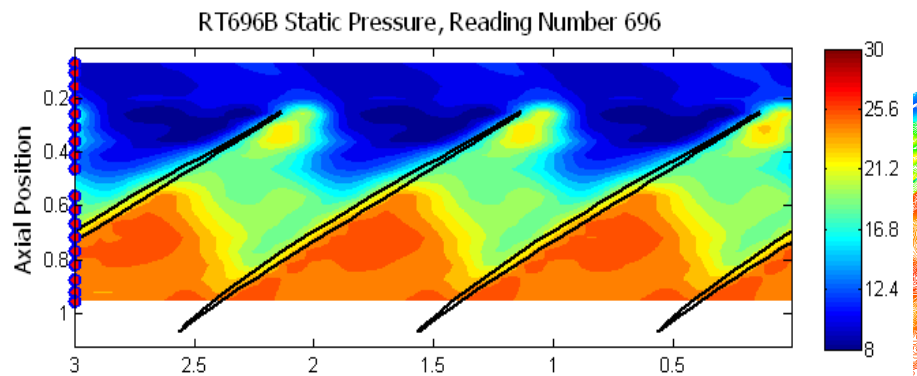

Measurement

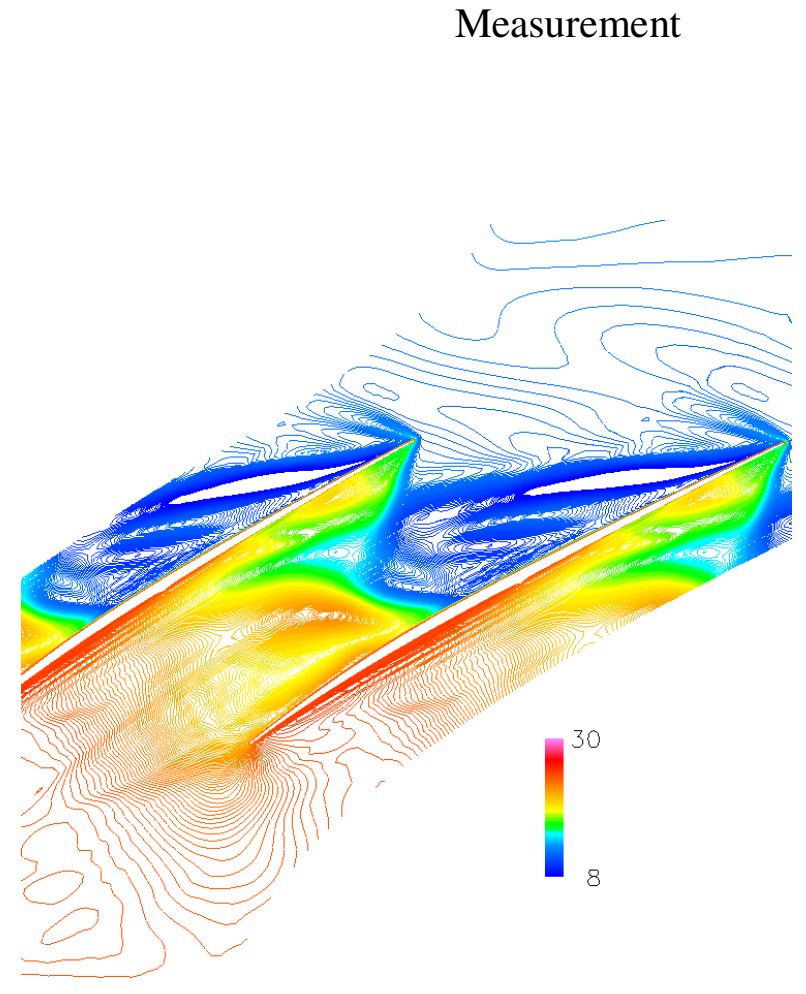

\section{LES}

Figure 4c: Comparison of casing static pressure distribution at near stall (Point $\mathrm{C}$ in Figure 3).
\end{abstract}

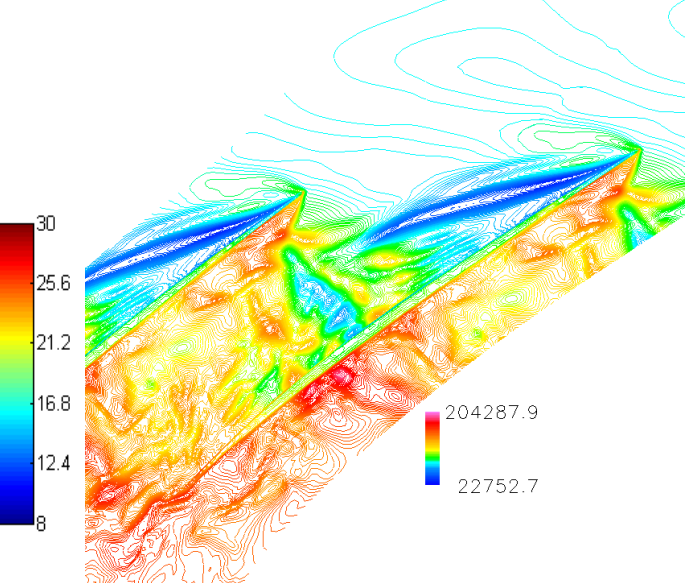

Time 1
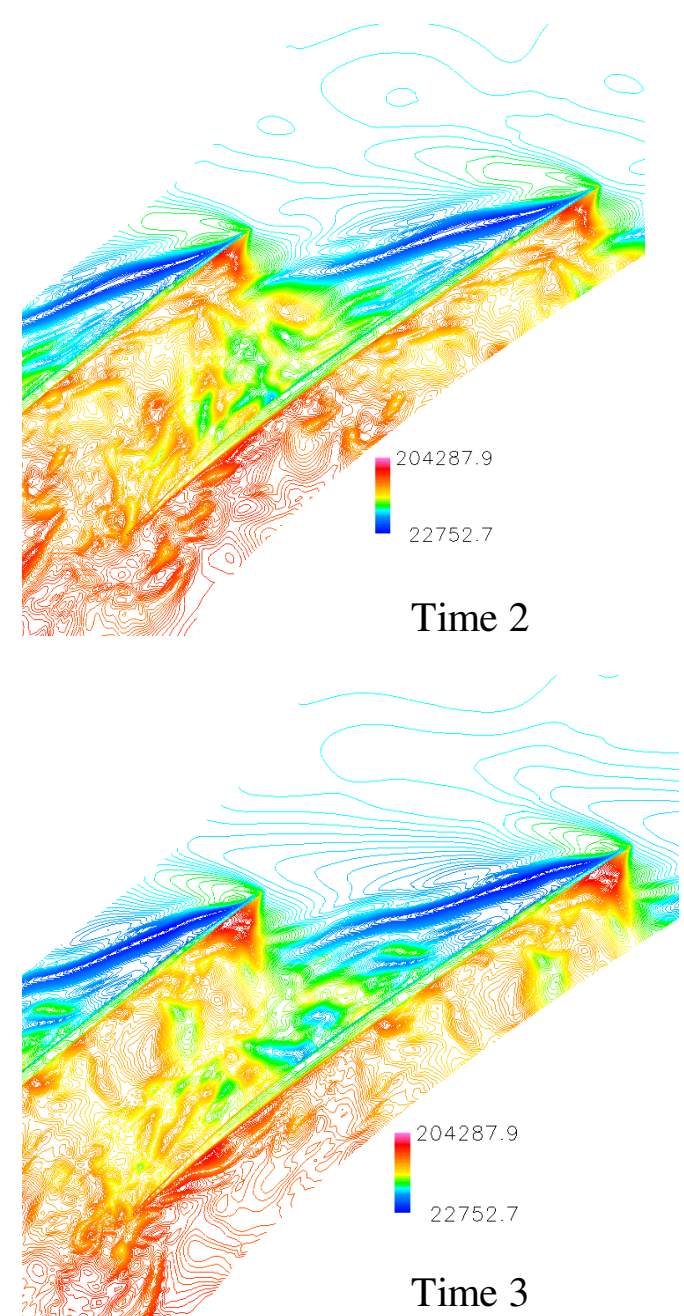

Figure 5: Changes in static pressure at rotor tip, near stall, Point $D$ in Figure 3. 

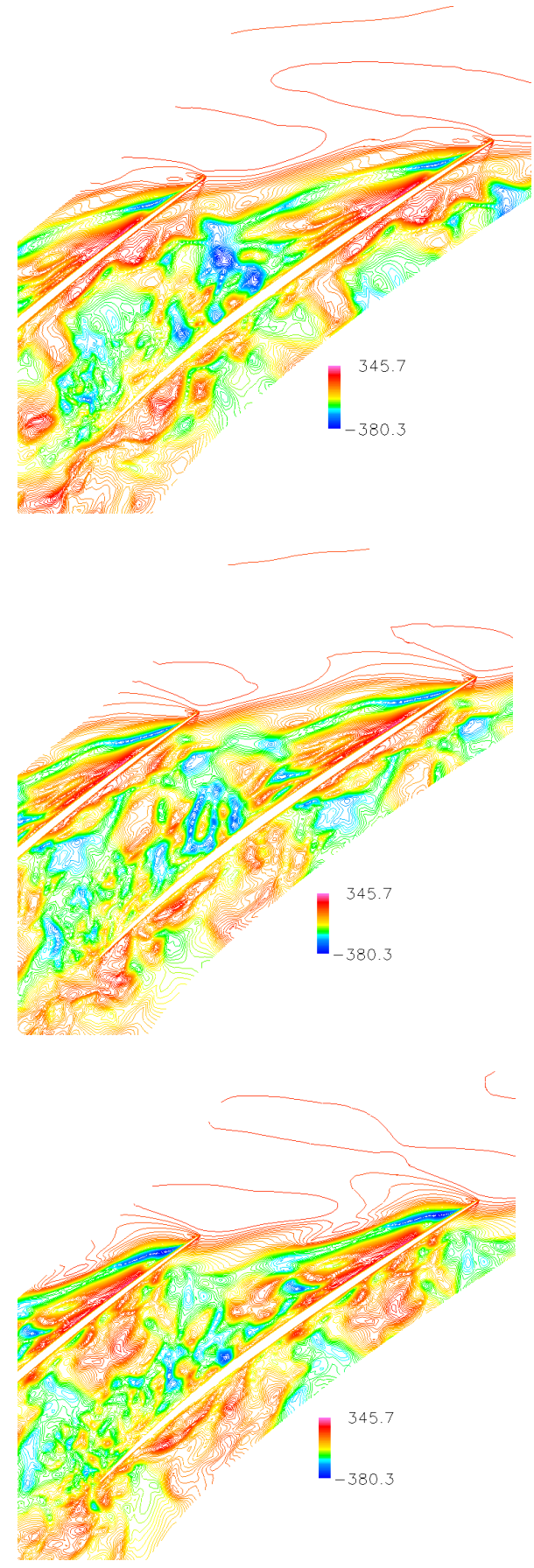

Figure 6: Changes in axial velocity at rotor tip, near stall, Point $D$ in Figure 3.

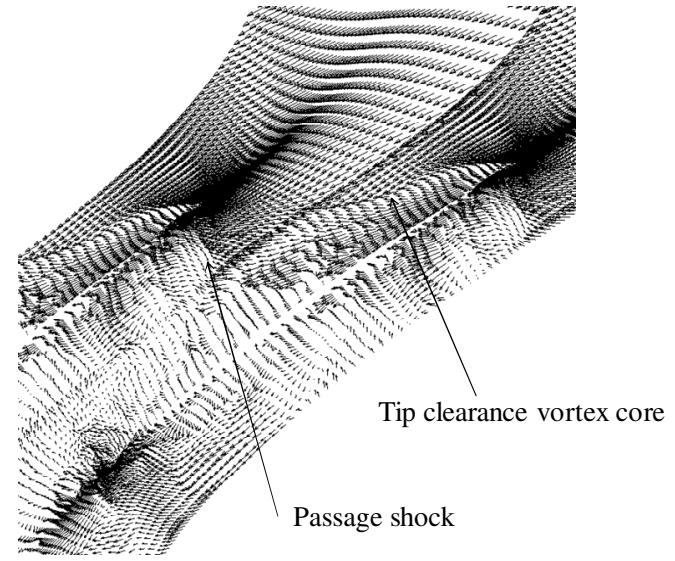

Figure 7: Instantaneous velocity vectors at rotor tip, near stall condition, Point D in Figure 3.

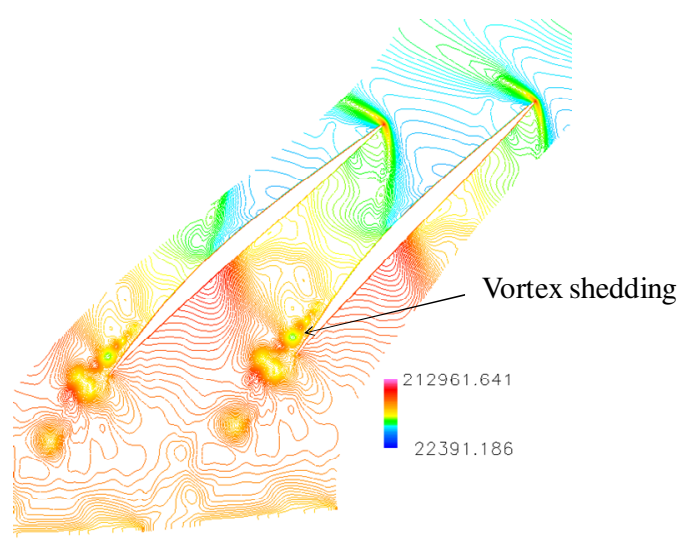

Figure 8: Instantaneous pressure distribution at $40 \%$ span, near stall, Point $D$ in Figure 3. 


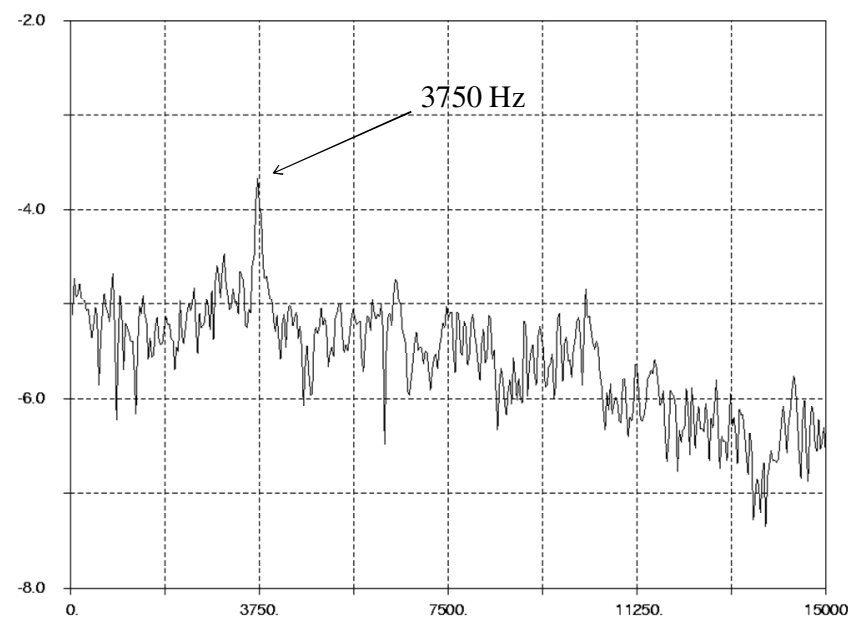

Figure 9: Spectrum from casing pressure, single passage LES, near stall, Point $D$ in Figure 3.

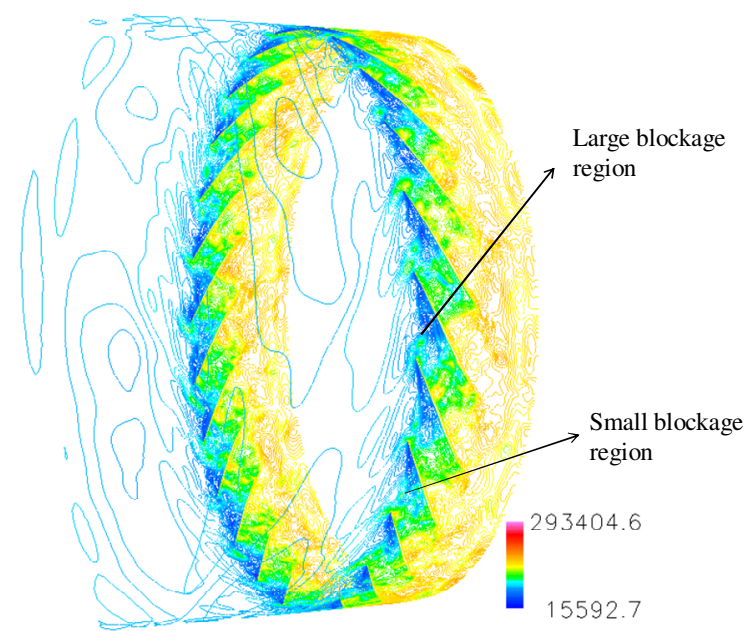

Figure 10: Instantaneous pressure distribution at rotor tip, near stall, Point D in Figure 3.

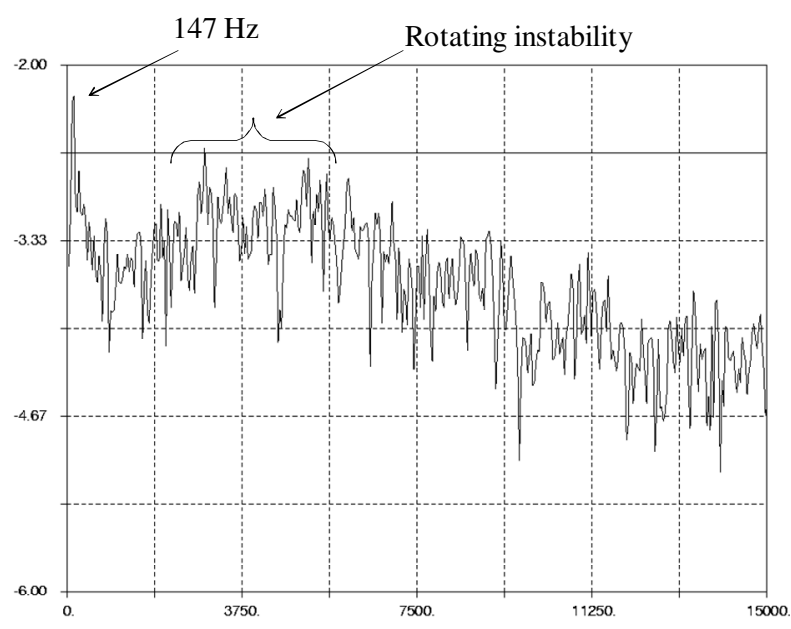

Figure 11: Spectrum from casing pressure, full annulus analysis, near stall, Point $D$ in Figure 3. 

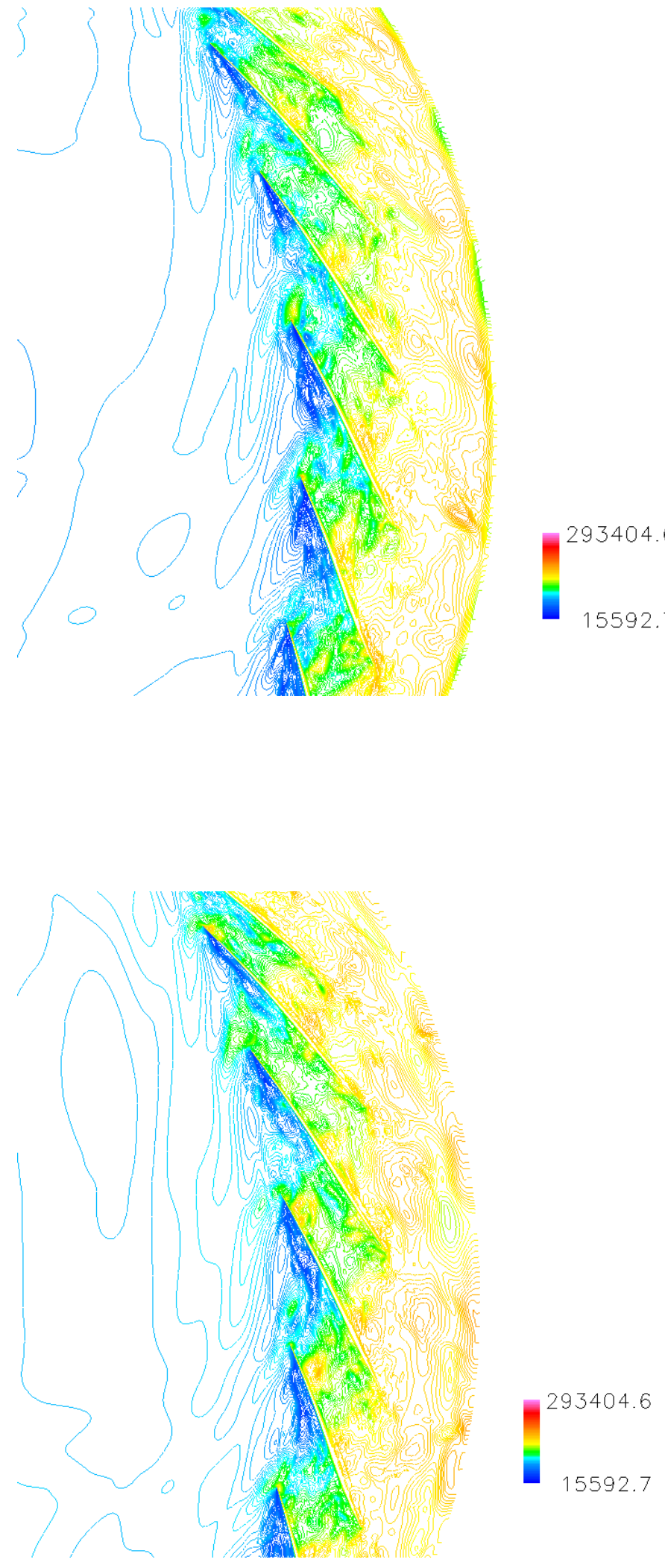

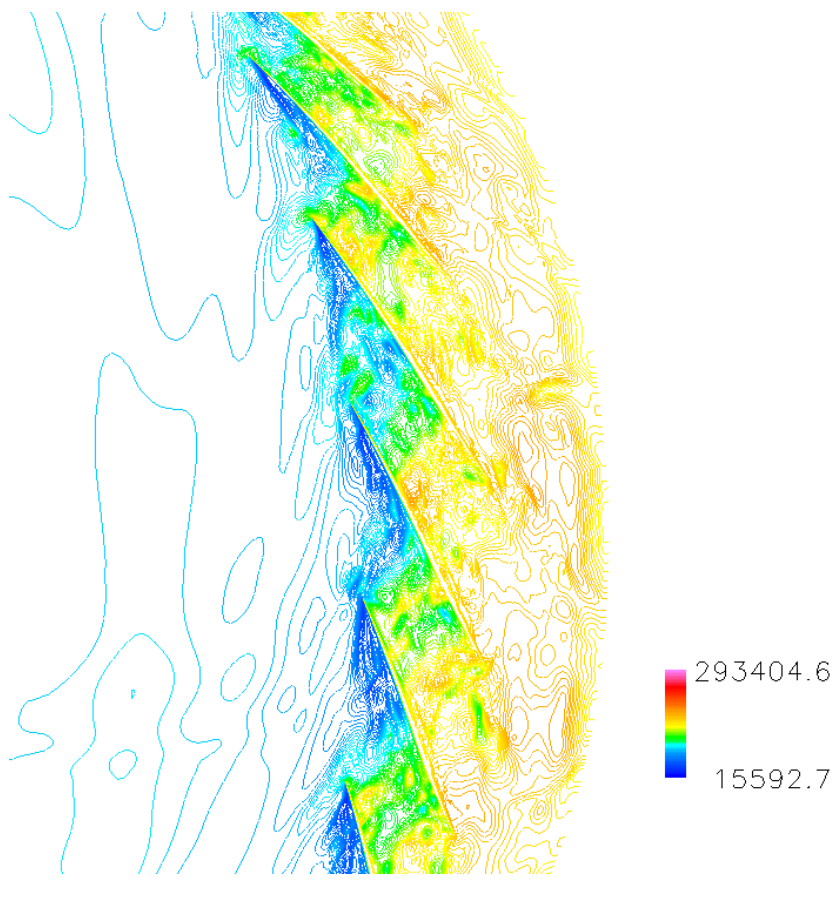

Figure 12: Changes in static pressure at rotor tip, near stall, Point $D$ in Figure 3. 

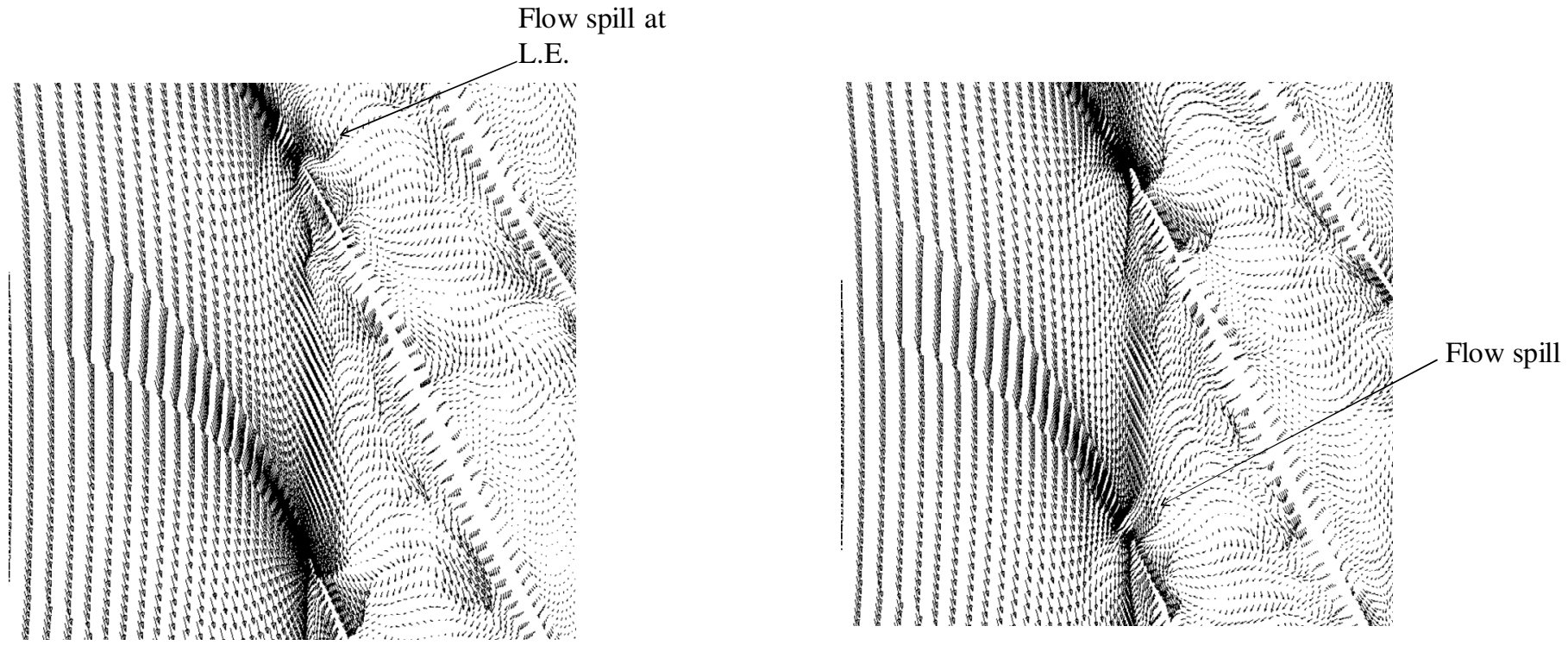

Figure 13: Changes in velocity vector at rotor tip, near stall, Point D in Figure 3.

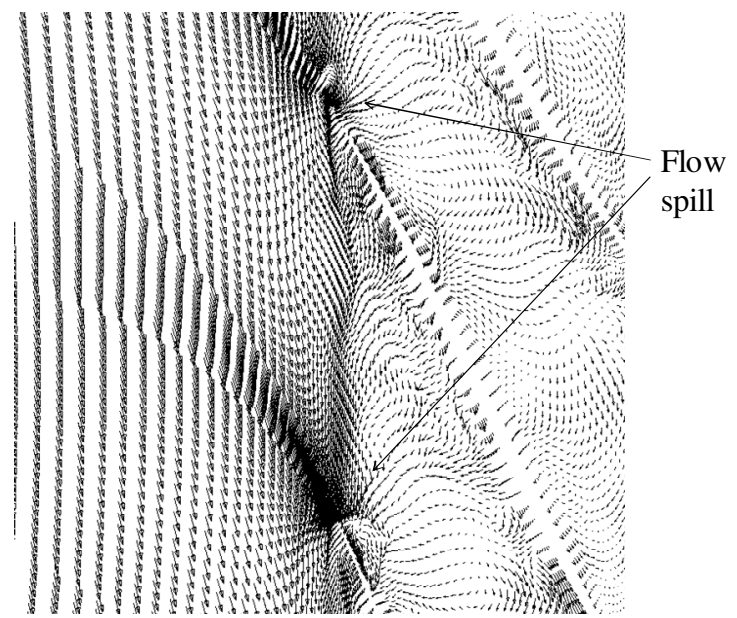


